\title{
DER ÖFFENTLICHE NOTAR IM GEFLECHT DER PREUSSISCHEN SCHREIBER DES FRÜHEN 15. JAHRHUNDERTS
}

KEYWORDS

Prussia; notary public; agnatic and cognatic relations; priesthood; individual career; administrative reform

m die Vesperzeit des 20. Oktobers des Jahres 1400 versammelten sich auf
der Marienburg im Wohngemach von Hochmeister Konrad von Jungin-
gen der Dompropst von Marienwerder, Johannes Rymann, der Pfarrer von Thiergart, Paul Strik, und dessen Brüder Bernhard und Peter - der eine Schreiber des Danziger Komturs, der andere im Schreibdienst des Vogtes von Dirschau -, um vor dem ebenfalls anwesenden öffentlichen Notar und späteren Ordensprokurator bei der Kurie, Peter von Wormditt, einen unter ihnen ausgehandelten Vergleich zu beurkunden. ${ }^{1}$ Danach verweigerte der Dompropst von Rechts wegen zwar die Rückzahlung der von dem verstorbenen pomesanischen Domherrn und Pfarrer von Elbing, Nikolaus Holland, von seinen Verwandten, den Gebrüdern Strik, im Namen der Elbinger Pfarrkirche geborgten Summe Geldes. Johannes Rymann übereignete den Gebrüdern aber aus besonderer Gunst, wie es heißt, zwei silberne Gefäße.

Dieses Beispiel aus dem Jahre 1400 wirft nicht nur ein Schlaglicht auf die Art und Weise, wie Schreiber im Ordensland Preußen beruflich und persönlich miteinander verknüpft waren, sondern auch auf die in den Quellen nur schwer fassbaren kognatischen Verwandtschaftsverhältnisse untereinander und nach außen.

Im ersten Schritt soll deshalb versucht werden, der Bedeutung der Verwandtschaftslinien, insbesondere der weiblichen, für die Verankerung der Schreiber im preußischen Ordensland nahe zu kommen.

1 Geheimes Staatsarchiv Preußischer Kulturbesitz (weiterhin: GStA PK), XX. HA, Pergamenturkunden, Schiebl. L, Nr. 28. 
Aufbauend auf den Untersuchungen insbesondere von Methner ${ }^{2}$, Thumser ${ }^{3}$, Tandecki ${ }^{4}$ und Radosław Biskup 5 , geht es im zweiten Schritt darum, die sonstigen Verbindungen der Schreiber untereinander weiter auszugestalten. Hierbei und zugleich als Quintessenz dürfte die Beantwortung von Fragen nach möglichen Hierarchien, die sich auf der Grundlage von Befähigungen oder Weihegraden von Schreibern, herausgebildet haben, besonders reizvoll sein.

\section{VERWANDTSCHAFTSLINIEN}

Dem soeben vorgestellten Notariatsinstrument Peters von Wormditt ist zu entnehmen, dass der Pfarrer des Kirchspiels Thiergart im östlichen Marienburger Werder, Paul Strik, und seine Brüder, die Schreiber Bernhard und Peter, mit Nikolaus Holland über die weibliche Linie verwandt waren. Nikolaus Holland, der in der Nacht des 20. Januar 1398 inmitten der Vorbereitung einer Predigt gestorbenen war $^{6}$, ist Ordensforschern kein Unbekannter; denn von 1395 bis 1397 hatte er als Kaplan des Hochmeisters Konrad von Jungingen die Leitung der hochmeisterlichen Kanzlei auf der Marienburg inne. Danach ist er bis zu seinem Tode als Pfarrer der Elbinger Stadtpfarrkirche St. Nikolai und pomesanischer Domherr belegt. Als sicher gilt die Personengleichheit mit dem Prager Studenten Nicolaus de Hollant Prutenus, der Ende Mai 1371 seine Ausbildung an der Prager Universität mit einem Bakkalaureat an der artistischen Fakultät abgeschlossen hatte. Mit Vorsicht behandelt die Forschung hingegen die Gleichsetzungen des zwischen

2 A. Methner, Die Danziger Stadtschreiber bis 1650, Danziger familiengeschichtliche Beiträge 1 (1929) (Neudr. Sonderschriften des Vereins für Familienforschung in Ost- und Westpreußen 60, Hamburg 1988), S. 27-39.

3 M. Thumser, Private Briefkonzepte aus dem Nachlaß des Deutschordenssekretärs Liborius Naker († 1502/1503), Archiv für Diplomatik 43 (1997), S. 413-454; und ders., Schriftlichkeit in der Spätzeit der preußischen Deutschordensherrschaft. Kanzleitätigkeit und Aufzeichnungen des hochmeisterlichen Sekretärs Liborius Naker (1502/1503), in: Schriftkultur und Landesgeschichte. Studien zum südlichen Ostseeraum vom 12. bis zum 16. Jahrhundert, hrsg. v. M. Thumser (Mitteldeutsche Forschungen 115), Köln-Weimar-Wien 1997, S. 155-218, hier 156, 158, 161.

4 J. Tandecki, Die Stadtschreiber und ihre Rolle bei der Vereinheitlichung der Arbeitsformen der städtischen Kanzleien in Preußen, in: Die Rolle der Stadtgemeinden und bürgerlichen Genossenschaften im Hanseraum in der Entwicklung und Vermittlung des gesellschaftlichen und kulturellen Gedankengutes im Spätmittelalter, hrsg. v. dems., Toruń 2000, S. 117-131, hier 129 f.

5 R. Biskup, Pisarze i notariusze biskupów sambijkich w XV i XVI wieku (do 1525 roku). Uwagi o pochoodzeniu, wykstatceniu i kariere, in: Kanzelaria wielkich mistrzów i polska kancelaria królewska wXV wieku, hrsg. v. J. Trupinda, Malbork 2006, S. 13-36.

6 M. Glauert, Das Domkapitel von Pomesanien (1284-1527) (Prussia Sacra 1), Toruń 2003, S. 532. 
1355 und 1360 belegten Priesterbruders Nicolaus de Hollandia und die des ebenfalls zu 1360 als Visitator nachgewiesenen Elbinger Pfarrers Nikolaus. Bei dem zu 1383 als Zeuge einer urkundlichen Beglaubigung hinzugezogenen Pfarrer von Preußisch Holland, Nikolaus, dürfte es sich indes nur um eine gleichnamige Person gehandelt haben. ${ }^{7}$ Sonst wäre die Anführung der bedeutenderen Pfarrstelle in Elbing bei der Zeugennennung zu erwarten gewesen.

Von den im Notariatsinstrument genannten Verwandten des Elbinger Pfarrers und pomesanischen Domherrn Nikolaus, den Gebrüdern Strik, ist von Paul, dem vermutlich ältesten Bruder, lediglich die Tatsache bekannt, dass er Pfarrer von Thiergart war. Aufgrund ihrer Schreibertätigkeit sind die beiden anderen Brüder, Peter und Bernhard, besser belegt. Da beide am 18. September 1387 vor der artistischen Fakultät der Prager Universität ihr Bakkalaureatsexamen unter ihrer Herkunftsangabe de Dyrsovia abgelegt hatten ${ }^{8}$, dürfte Dirschau als Herkunftsort der Familie Strik sicher sein. Peter Strik war von 1399 bis 1405 als Schreiber des Vogtes von Dirschau tätig. Ende April 1405 ist Peter im Umkreis des hochmeisterlichen Notars Johannes Lichtenwald, der vom Schreibdienst des litauischen Großfürsten Witold in den des Hochmeisters übergewechselt war, nachweisbar. Danach scheint Peter in den Dienst des Obersten Marschalls getreten zu sein, falls Petrus, der zum 12. Juli 1406 belegte Schreiber des Marschalls mit Peter Strik personengleich war, wie Mario Glauert vermutet. ${ }^{9}$

Eine steile Karriere scheint Peters Bruder Bernhard angestrebt zu haben, wie seine Immatrikulation in die juristische Fakultät der Prager Universität von 1388 vermuten lässt. ${ }^{10}$ Bernhards Rechtskenntnisse waren für seine Kanzleitätigkeiten sicherlich von großem Vorteil, sodass er seinen zweiten nachweisbaren Schreiberposten bereits in der Kanzlei des Hochmeisters Heinrich von Plauen innehatte. Bernhard erscheint nämlich zusammen mit dem Schreiber Ruland ${ }^{11}$, dem mutmaßlichen Nachfolger seines Bruders Peter beim Vogt von Dirschau, unter den Zeugen einer zu Marienburg datierten Urkunde vom 30. April 1411. ${ }^{12}$ Michael Küchmeister, Plauens Amtsnachfolger, beschäftigte Bernhard in seiner hochmeisterlichen Kanzlei weiter. Unter dem Hochmeisterkaplan Gregor von Bischofswerder ${ }^{13}$ war

7 Glauert (wie Anm. 6), S. $532 \mathrm{f}$.

8 M. Perlbach, Prussia scholastica. Die Ost- und Westpreussen auf den mittelalterlichen Universitäten (Monumenta historiae Warmiensis 6, 3), Braunsberg 1895, S. 14.

9 Glauert (wie Anm. 6), S. 534, Anm. 1174.

10 Perlbach (wie Anm. 8), S. 20.

11 Das Marienburger Tresslerbuch der Jahre 1399-1409, hrsg. v. [E.] Joachim, Königsberg 1896 (Neudr. Bremerhaven 1973); S. 376, Z. 8; Glauert (wie Anm. 6), S. 534, Anm. 1175.

12 GStA PK, XX. HA, Pergamenturkunden, Schiebl. XXIV, Nr. 9.

13 B. Jähnig, Hochmeisterkaplan und Hochmeisterkanzler. Die Leiter der Hochmeisterkanzlei in Marienburg 1309-1457, in: Kancerlarie Krzyżackie. Stan badań i perspektywy badawcze, hrsg. 
Bernhard in der Marienburger Kanzlei als erster Schreiber ${ }^{14}$ tätig. Aus dieser Zeit stammen die Nachrichten, dass Bernhard Strik Diakon des Leslauer Bistums war und dass der Hochmeister ihn als Prokurator und Syndikus verwendete, wie z. B. bei einer Appellation gegen ein Urteil der Bischöfe von Leslau und Posen von 1417 April 19 $9^{15}$. Offenbar noch Größeres hatte der Hochmeister mit Bernhard vor, denn er providierte ihn am 11. März 1417 zum Priester ${ }^{16}$, sodass Bernhard seine Priesterweihe zwischen dem 4. und 18. November 1417 empfangen konnte ${ }^{17}$. Bereits am 27. November 1417 hatte Bernhard die Pfarrstelle in Heilsberg erhalten ${ }^{18}$, auf die er jedoch wenige Monate später zu Gunsten des ermländischen Offizials Jakob Magni verzichten musste. ${ }^{19}$ Bernhard ließ sich mit der Pfarrei von Rößel abfinden, falls der in einer Urkunde von 1426 Mai $28^{20}$ unter den Zeugen genannte gleichnamige Rößeler Pfarrer mit ihm personengleich war.

Die Schreiberberufe von Peter und Bernhard Strik erhärten übrigens den von Janusz Tandecki geäußerten Verdacht, wonach es auch im Preußenland Familien gegeben habe, die den Schreiberberuf und die damit verbundenen Fertigkeiten von Generation zu Generation weiter gaben. ${ }^{21}$

Der Vertreter der Gegenpartei des Vergleichs, der am 20. Oktober 1400 im Wohngemach des Hochmeisters ausgehandelte wurde, nämlich der Domherr und nachmalige pomesanische Bischof Johann Rymann, stammte aus der Diözese Pomesanien. Glauert identifiziert ihn zu Recht mit Ioan[nes] Rymanni de Crystburg, der Ende September 1382 vor der Prager Artistenfakultät sein Bakkalaureatsex-

v. J. Trupinda, Malbork 2002, S. 149-166, hier 156.

14 Beispiele: Regesta historico-diplomatica Ordinis S. Mariae Theutonicorum 1198-1525, bearb. v. E. Joachim, hrsg. v. W. Hubatsch, Pars I: Regesten zum Ordensbriefarchiv, Vol. 1-3; Pars II: Regesten der Pergament-Urkunden aus der Zeit des Deutschen Ordens, Göttingen 1948-1973, hier Pars II, Nr. 1825 zu 1415 März 2; und Ksiega komturstwa Gdańskiego, hrsg. v. K. Ciesielska, I. Janosz-Biskupowa (Towarzystwo Naukowe w Toruniu, Fontes 70), Warszawa u. a. 1985, Nr. 83 zu 1417 Nov. 4.

15 GStA PK, XX. HA, OBA Nr. 2502, dort (recto) als diaconus Wlad(islaviensis) dyoc(esis) tituliert, von Koeppen, Die Berichte der Generalprokuratoren des Deutschen Ordens an der Kurie, Bd. 2: Peter von Wormditt (1403-1419), bearb. v. H. Koeppen (Veröffentlichungen der Niedersächsischen Archivverwaltung 13), Göttingen 1960, Nr. 204, Anm. 5, als decanus gelesen und ihm darin folgend Glauert (wie Anm. 6), S. 535, Anm. 1178.

16 GStA PK, XX. HA, OF 281, S. 173.

17 In einer hochmeisterlichen Urkunde von 1417 Nov. 18 erscheint Berhard Stryg in der Zeugenreihe vor den Schreibern mit dem Titel ,Herr', Regesta historico-diplomatica, Pars II (wie Anm. 14), Nr. 1891; bei Glauert (wie Anm. 6), S. 535 Anm. 1178 irrtümlich JH II, 1819 statt JH II, 1891.

18 Die Berichte (wie Anm. 15), Nr. 241, Anm. 3.

19 Die Berichte (wie Anm. 15), Nr. 259.

20 Regesta historico-diplomatica, Pars II (wie Anm. 14), Nr. 2243.

21 Tandecki (wie Anm. 4), S. 129. 
amen abgelegt hatte und der 1387 die Prager Universität als doctor decretorum verließ.22 Bei ihm lassen sich weder verwandtschaftliche Beziehungen zu den Gebrüdern Strik oder zu Nikolaus Holland noch zu den vielen im Preußenland vorkommenden Trägern des Nachnamens, Rymann' nachweisen.

Immerhin geben die soeben skizzierten Lebensläufe genug Anlass zur Behauptung, dass die am Vergleich beteiligten Personen einander gut bekannt waren. In diese Richtung deutet auch die Gunstbezeugung in Gestalt der beiden Silbergefäße, die Dr. Johann Rymann den Gebüdern Strik überlassen hatte.

Der für die Beglaubigung des Vergleichs hinzugezogene öffentliche Notar Peter von Wormditt war zu dem Zeitpunkt erster Schreiber des Hochmeisters. ${ }^{23}$

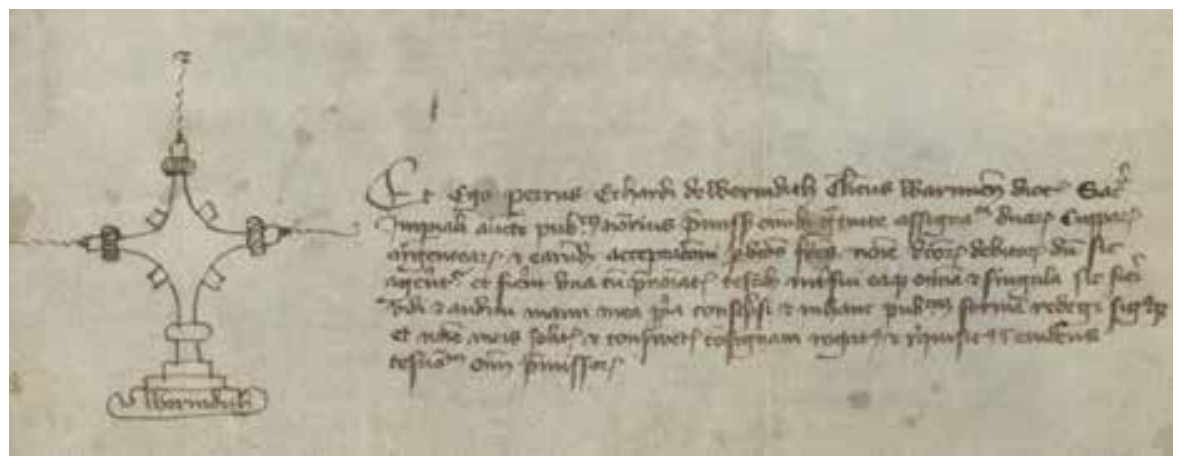

Abb. ( ) GStA PK (Signet und Notarsunterschrift Peters von Wormditt, GStA PK, XX. HA, Pergamenturkunden, Schiebl. L, Nr. 28)

Mittels seiner Notarsunterschrift weist sich Peter als Sohn des Echard von Wormditt und als Kleriker der ermländischen Diözese aus. Eine Verwandtschaftsbeziehung zu Walther, Sohn des Echard aus Bunzlau, der zwischen 1377 und 1402 als Stadtschreiber von Thorn belegt ist ${ }^{24}$, lässt sich zwar nicht nachweisen. Neben dem gleichlautenden Vaternamen und der gemeinsamen Schreibertätigkeit fällt aber auf, dass Schlesien als Herkunftslandschaft beider Schreiberfamilien wahr-

22 Glauert (wie Anm. 6), S. 480.

23 M. Armgart, Die Handfesten des preußischen Oberlandes bis 1410 und ibre Aussteller (Veröffentlichungen aus den Archiven Preußischer Kulturbesitz, Beiheft 2), Köln-Weimar-Wien 1995, S. 253-259, hier 254.

24 Ksiegri szosu i wykazy obciązeń miesczkańców Starego Miasta Torunia z lat 1394-1435, hrsg. v. K. Mikulski, J. Tandecki, A. Czacharowski (Quellen zur Geschichte des mittelalterlichen Thorn 1), Toruń 2002, S. xxxii f. 
scheinlich ist: Bei Walther ist das schlesische Bunzlau Bestandteil des Namens und bei Peter die schlesischen Pflanzstadt Wormditt.

Gesichert ist dagegen Peter von Wormditts Verwandtschaft mit der Elbinger Bürgerin Katke Kreft. Katke war in erster Ehe mit dem Elbinger Ratmann Godeke von Alen verbunden. Mit ihm hatte sie die Söhne Peter, Andreas und Godeke. Danach war sie mit dem Lokator des Elbinger Vorwerks Zwenhusen, Johannes Kreft, verheiratet. Ihre gemeinsame Tochter Katke d. J. Kreft war mit dem Elbinger Ratmann Johann d. J. Hervord verehelicht, der 1410 bei Tannenberg gefallen ist. ${ }^{25}$ Verwandte, wie z. B. der Ratmann Nikolaus Thomas und die Familie Grudentz, hatte Peter von Wormditt auch unter der Danziger und der Königsberger Bürgerschaft. Johannes Rex, ein Schwestersohn Peters, war überdies Domherr in Frauenburg. ${ }^{26}$

\section{Sonstige Schreiber-Verbindungen Und SChreiberhierarchien}

Wenn im Folgenden die Verbindungen von Schreibern untereinander kurz beleuchtet werden sollen, so sind vor allem Bekanntschaften und andere Arten künstlicher Verwandtschaft, aber auch wechselseitige Abhängigkeiten auf gleicher oder auf hierarchischer Ebene, gemeint.

Die Erkenntnis, dass Schreiber sich im Preußenland zu Bruderschaften zusammengeschlossen hatten, ist zwar nicht neu, aber kaum belegt. Gesichert ist nach derzeitigem Kenntnisstand lediglich die Stiftung der Dorothenkapelle zu Danzig durch die dortigen Schreiber und Kleriker, weil Papst Urban VI. für die Unterstützung des Kapellenbaus am 2. Mai 1382 in Rom eine Ablassbulle ausgefertigt hat. ${ }^{27}$ Über die Mitglieder der seit 1369 nachweisbaren Bruderschaft gab das heute leider verschollene Totenbuch Auskunft. Paul Simson, der das Buch noch vor dem Ersten Weltkrieg eingesehen hatte, teilt daraus lediglich den Namen des 1368 amtierenden Pfarrers von St. Marien, Johannes von Gilgenburg, mit. ${ }^{28}$

25 Das Elbinger Kriegsbuch (1383-1409). Rechnungen für städtische Aufgebote, bearb. v. D. Heckmann u. Mitbearb. v. K. Kwiatkowski (Veröffentlichungen aus den Archiven Preußischer Kulturbesitz 68), Köln-Weimar-Wien 2013, S. 242 s. n. Kreft, Johanes. Bei der von Peter von Wormditt genannten Katke Krewissynne vom Elwinge handelt es sich nicht um Katke d. J., wie Nieborowski und Köppen vermuten, sondern wegen des den Witwenstand anzeigenden Suffixes -ynne um ihre Mutter, die noch zur Zeit des Schreibens Peters von Wormditt an den Hochmeister von 1418 Dez. 21 lebte, s. Die Berichte (wie Anm. 15), Nr. 301.

26 P. Nieborowski, Der Deutsche Orden und Polen in der Zeit des größten Konfliktes, Breslau ${ }^{2} 1924$, S. 8 f.

27 P. Simson Geschichte der Stadt Danzig, Bd. 1-4, Danzig 1913, hier Bd. 4, Nr. 104.

28 Simson (wie Anm. 17), Bd. 1, S. 85, 87. 
Ungewöhnlich erscheint das Dorotheenpatrozinium, gelten doch als Schutzheilige für die Notare die Evangelisten Johannes und Markus sowie Luzia von Syrakus. Stiftungen zu Ehren des Evangelisten Johannes, wie z. B. die von Bischof Berthold von Pomesanien vom 27. Dezember $1342^{29}$, legen vielleicht einen noch zu verfolgenden Pfad frei, der in diesem Zusammenhang freilich nur aufscheinen soll.

Schon das eingangs vorgestellte Notariatsinstrument deutet an, dass das Schreibpersonal im Ordensland Preußen untereinander gut bekannt war. Der Besuch ein und derselben Universität konnte von Vorteil sein. Es mag dem $\mathrm{Zu}$ fall geschuldet sein, dass die beiden Absolventen der Prager Universität Peter von Wormditt und Peter Strik im Schreibdienst des Obersten Marschalls nachweisbar sind, der eine von 1396 bis $1399^{30}$, der andere im Jahre 1406. ${ }^{31}$ Wer sich jedoch die Hochschulausbildung der Schreiber beispielsweise unter dem Hochmeisterkaplan Arnold Stapel (1397 Sept. 20 - 1402 Aug. 30) ${ }^{32}$ anschaut, der glaubt wohl kaum noch an Zufälligkeiten; denn unschwer ist das Bemühen des Kaplans zu erkennen, die hochmeisterliche Kanzlei mit Leuten zu besetzen, die wie er in Prag studiert hatten. In ähnlicher Weise umgab sich ein halbes Jahrhundert später der Hochmeisterkaplan und Wiener Hochschulabsolvent Andreas Santberg mit Schreibern, die ihre universitäre Ausbildung in Wien erhalten hatten. ${ }^{33}$ Zum „Prager"-Personal von Arnold Stapel gehörten zumindest Hoyke von Konyec und Peter von Wormditt. ${ }^{34}$ Auffallend ist zudem, dass Stapel anscheinend besonderen Wert auf die Beschäftigung von Schreibern legte, die wie er selbst ${ }^{35}$ als öffentliche Notare approbiert waren. Dies lässt sich an der Beschäftigung der öffentlichen Notare Hoyke von Konyec, Peter von Wormditt und Nikolaus Berger $^{36}$ leicht erkennen, die nacheinander als erste Schreiber in der Kanzlei unter Stapel tätig waren. Dort regelten allem Anschein nach die öffentlichen Notare das hochmeisterliche Registerwesen nach dem Vorbild der bischöflichen und domkapitularischen Registraturen neu. Sie scheinen die größten Motoren und zugleich Träger und Verbreiter der allgemeinen Verwaltungsreform gewesen zu sein, die

29 Glauert (wie Anm. 6), S. 84.

30 Die Berichte (wie Anm. 15), S. 29 f., Anm. 15.

31 Glauert (wie Anm. 6), S. 534, Anm. 1175.

32 Armgart (wie Anm. 23), S. 172-178.

33 Die Chronik vom Bund und Vereinigung wider Gewalt und Unrecht des Hochmeisterkaplans Andreas Santberg zur Vorgeschichte des Dreizehnjährigen Krieges in Preußen (1450-1454), bearb. v. D. Heckmann u. Mitwirkung v. M.-L. Heckmann (Einzelschriften der Historischen Kommission für ost- und westpreußische Landesforschung 27), Marburg 2007, S. 11.

34 Armgart (wie Anm. 23), S. 256.

35 Armgart (wie Anm. 23), S. 175.

$36 \mathrm{Zu}$ Berger s. Armgart (wie Anm. 23), S. 259-263. 
Hochmeister Winrich von Kniprode mit der im Jahre 1380 in Gang gesetzten allgemeinen Landvermessung angestoßen hatte. ${ }^{37}$

Die Wirkungder öffentlichen Notare als Träger der Verwaltungsreform kommt auch in anderen Schreibstuben des Preußenlandes zum Zuge: Im Marschallamt lässt sich seit den 90er Jahren des 14. Jahrhunderts erstmals eine sorgfältige Registrierung der dort ausgestellten Urkunden beobachten, was zu einem guten Teil dem Verdienst Peters von Wormditt zuzumessen ist, der - wie schon gesagt - von 1396 bis $1399 \mathrm{im}$ Schreibdienst des Obersten Marschalls nachweisbar ist. ${ }^{38}$ Wenige Zeit später begegnet in der vom Marschallamt abhängigen Königsberger Großschäfferei der öffentliche Notar Johannes Schoenaw. Er war zuvor Schreiber seiner Heimatstadt Graudenz. Schoenaw diente dem Großschäffer zwischen den Jahren 1404 und 1407 und übernahm danach den Posten des Stadtschreibers von Kulm, den er bis 1430 bekleidete. ${ }^{39}$ In Elbing löste der öffentliche Notar Bartholomeus Stume 1408 Johann Birsmit im Amt des Stadtschreibers ab. ${ }^{40}$ Darin ist Bartholomeus noch 1417 nachweisbar. ${ }^{41}$ Die Altstadt Thorn beschäftigte in den Jahren 1409 und 1410 den öffentlichen Notar Franz Slauser als Stadtschreiber. Und der Danziger Rat bestellte im Jahre 1423 den öffentlichen Notar Konrad Bitschin zum Stadtschreiber. ${ }^{42}$ In den mit der Marienburger Zentrale eng verbundenen Schreibstuben des Bischofs und des Domkapitels von Pomesanien wirkten in der Sattelzeit zwischen dem 14. und 15. Jahrhundert der öffentliche Notar Arnold von Riesenburg ${ }^{43}$ als Schreiber von Bischof Johann Mönch, der selber öffentlicher Notar war. ${ }^{44}$ In der samländischen Kanzlei zu Fischhausen waren von 1410 bis 1421 nacheinander die öffentlichen Notare Nikolaus Becker aus Danzig und der Westfale Lampertus de Vico tätig. ${ }^{45}$ Im Ermland ist der öffentliche Notar Kaspar Schuwenpflug zu den Jahren 1398 und 1399 als Schreiber des Bischofs Heinrich

37 D. Heckmann, Ferment in der Verwaltung: das öffentliche Notariat im Deutschordensland PreuBen, Archivalische Zeitschrift 93 (2012), S. 204-220.

38 Heckmann (wie Anm. 37), S. 220.

39 D. Heckmann, Der öffentliche Notar im Ordensland Preußen im Spiegel von Rechtsrezeption und Kanzleipraxis, Preußenland 47 (2009), S. 37-56, hier 49 f.

40 Tandecki (wie Anm. 4), S. 122; Z. H. Nowak, O sposobach werbowania intelektualistów do zakonu krzyżackiego w Prusach w pierwszej potowie XV wieku, Zapiski Historyczne 45 (1980), 2, S. 101-106, hier 104.

41 Das Elbinger Kriegsbuch (wie Anm. 15), S. 29 f.

42 A. Methener, Conrad Bitschin als Danziger Stadtschreiber, Zeitschrift des Westpreußischen Geschichtsvereins 69 (1929), S. 69-83, hier 74.

43 Glauert (wie Anm. 6), S. 404-406.

44 Heckmann, Der öffentliche Notar (wie Anm. 39), S. 48.

45 E. Weise, Das Urkundenwesen der Bischöfe von Samland, Altpreußische Monatsschrift 59 (1922), S. 1-48, 157-209, hier 16. 
Sorbom nachweisbar. ${ }^{46}$ Der aus der in Passenheim ansässigen Lokatorenfamilie stammende Schuwenpflug sollte später als Diplomat unter dem Generalprokurator Peter von Wormditt und als Bischof von Ösel-Wiek seinen Aufstieg in der Ordenshierarchie machen. ${ }^{47}$

\section{QUINTESSENZ}

Die bisherigen Ausführungen mögen den Eindruck hinterlassen haben, als stünden die öffentlichen Notare um die Wende vom 14. zum 15. Jahrhundert an der Spitze der Hierarchie der preußischen Schreiber. Indes warnen Gegenbeispiele vor vorschnellen Urteilen. So liegen keine Kenntnisse darüber vor, dass die um 1400 tätigen Schreiber Johann Crolow ${ }^{48}$ und Michael Kesker ${ }^{49}$ aus der Rechtstadt und aus der Jungstadt Danzig oder Johann Frederici ${ }^{50}$ von der Altstadt Königsberg ebenfalls die Befähigung zum öffentlichen Notar hatten. Mit an Sicherheit grenzender Wahrscheinlichkeit war auch Johann Birsmit, der alte Stadtschreiber von Elbing, kein öffentlicher Notar. Er bezog wie sein Nachfolger Bartholomeus das gleiche Jahresgehalt in Höhe von $12 \mathrm{Mark}^{51}$ und war ihm so formal gleichgestellt. Der Umstand, dass Bartholomeus vor seinem Amtsantritt den Umbau des Schreiberhauses am Elbinger Rathaus offensichtlich auszuhandeln vermochte ${ }^{52}$, spricht allerdings für das Ansehen, das Bartholomeus als öffentlicher Notar genoss.

Trotz dieser soeben geäußerten Einwände lässt sich mit ziemlicher Sicherheit behaupten, dass die Verbindung von öffentlichem Notariat und Priestertum besonders karriereträchtig war: Peter von Wormditt war als Generalprokurator an der Kurie der einflussreichste Ordensdiplomat seiner Zeit, der Elbinger Bürgersohn Johann Mönch und der Passenheimer Lokatorensohn Kaspar Schuwenpflug

46 Codex Diplomaticus Warmiensis oder Regesten und Urkunden zur Geschichte Ermlands, Bd. 3, hrsg. v. C. P. Woelky, J. M. Saage (Monumenta Historiae Warmiensis 5), Mainz 1874, Nr. 336, 344.

47 B. Jähnig, Kaspar Schuwenpflug († 1423). 1418-1420 Koadjutor des Bischofs von Ösel-Wiek. 1420-1423 Bischof von Ösel-Wiek, in: Die Bischöfe des Heiligen Römischen Reiches 1198 bis 1448. Ein biographisches Lexikon, hrsg. v. E. Gatz unter Mitwirkung v. C. Brodkorb, Berlin 2001, S. $497 \mathrm{f}$.

48 Tandecki (wie Anm. 4), S. 120.

49 Ksiegi Mtodego Miasta Gdańska 1400-1455 [1458-1459], hrsg. v. K. Kopiński, P. Oliński (Towarzystwo Naukowe w Toruniu, Fontes 100), Toruń 2008, S. xxxi.

50 Tandecki (wie Anm. 4), S. 123.

51 Das Elbinger Kriegsbuch (wie Anm. 15) S. 28-31.

52 Das Elbinger Kriegsbuch (wie Anm. 15) S. 31. 
beendeten ihre Karrieren als Bischöfe. Der öffentliche Notar Arnold von Riesenburg brachte es immerhin bis zur Würde eines pomesanischen Domherrn.

Die skizzierten Verflechtungen trugen sicherlich in einem erklecklichen Maße dazu bei, dass um die Marienburg die schon der älteren Forschung bekannte Schreiberlandschaft entstehen konnte, in der sich eine Spielart des Ostmitteldeutschen als Amtssprache durchzusetzen vermochte. ${ }^{53}$

\section{SuMmary \\ The public notary in the network of Prussian scribes in the early $15^{\text {th }}$ century}

The article investigates the blood-relations between notable Prussian scribes, clergy and merchants, and demonstrates the importance of women in the shaping of family relations in late medieval Prussia. Individuals analyzed in the study include two scribes, Bernhard and Peter Strik, their brother Paul, parish priest of Thiergart, and Nikolaus Holland, cathedral provost of Marienverder as well as the public notary and future diplomat in service of the Teutonic Grand Masters, Peter of Wormditt and his kinship relations to various citizens of Elbling, Danzig and Königsberg. The combination of priesthood with the role of a public notary was a popular practice ensuring personal career in the Prussian society in the $15^{\text {th }}$ century. The evidence suggests that the network of Prussian scribes contributed to the formation of a kind of scribe-region with the vernacular ostmitteldeutsch as community language.

53 Das Grosse Ämterbuch des Deutschen Ordens, hrsg. v. W. Ziesemer (Veröffentlichung der Westpreußischen Provinzial-Verwaltung), Danzig 1921, S. xxii f. 